MATEC Web of Conferences 25,03012 (2015)

DOI: $10.1051 /$ matec conf/ 20152503012

(C) Owned by the authors, published by EDP Sciences, 2015

\title{
Applied Research of Enterprise Cost Control Based on Linear Programming
}

\author{
Shuo $\mathrm{Yu}$ \\ Finance Management Department, Liaoning Jianzhu Vocational University, Liaoyang, Liaoning, China
}

\begin{abstract}
As the economic globalization constantly advancing, the competition in the market economy has become even more intense, especially the growing nationalization in the competition among enterprises. The enterprise cost control is essential. Only when the enterprise cost control is within a reasonable range, the corresponding competitiveness of enterprises will be greatly enhanced, so the enterprise cost control is currently the strategy commanding point contended for by all parties. The linear programming is a calculation method to research the optimization of the objective function in the operational research under the relevant linear restrictive conditions. The linear programming is relatively mature and complete in the operational research, and it especially occupies a very important position in the economic field. The linear programming has clear steps and little computing complexity, which is helpful for dealing with the problem quickly. To transform the real problems into linear programming problems can demonstrate the impact of the various factors in the problems on the overall results, which can not only demonstrate the restrictive conditions well, but also determine the optimal solution of the objective result under such restrictive conditions. It requires accurately and actually transforming the problems, and determining the restriction factors and ultimate goals.

This paper researches the enterprise cost control through the linear programming model, and analyzes the restriction factors of the labor of enterprise production, raw materials, processing equipment, sales price, and other factors affecting the enterprise income, so as to obtain an enterprise cost control model based on the linear programming. This model can calculate rational production mode in the case of limited resources, and acquire optimal enterprise income. The production guiding program and scheduling arrangement of the enterprise can be obtained through calculation results, so as to provide scientific and effective guidance for the enterprise production. This paper adds the sensitivity analysis in the linear programming model, so as to learn about the stability of the enterprise cost control model based on linear programming through the sensitivity analysis, and verify the rationality of the model, and indicate the direction for the enterprise cost control. The calculation results of the model can provide a certain reference for the enterprise planning in the market economy environment, which have strong reference and practical significance in terms of the enterprise cost control.
\end{abstract}

Keywords: cost control; linear programming; sensitivity analysis

\section{INTRODUCTION}

The enterprise cost control is not only one of the most important issues to be addressed by the enterprise, but also a key factor to determine whether the enterprise can gain a competitive advantage in the intense market environment, which is also an inevitable requirement of the economic market competition. The enterprise cost control is a more complex issue, which is not just the reduction of the cost, but also a reasonable arrangement of the cost with the maximum benefit goals, so as to achieve the most optimal results in all aspects. Therefore, the enterprise cost control requires scientific research and rational optimization ${ }^{1]}$, so as to achieve the purposes of cost control and improvement of competitiveness.

The enterprise cost control is the most optimal allocation of resources, manpower and material resources. With limited resources and a number of practical restrictive conditions, the cost control is often not ideal, which requires reasonable arrangement by actu- al restrictive conditions, and currently with the lowest cost and maximum efficiency. Therefore, the solution to the enterprise cost control requires using the operational research based on the linear programming. The linear programming method is to transform the restrictive conditions in the problems into the decision variable equation of the objective function, of which the objective function is a linear function under the restriction of the decision variables ${ }^{[2]}$. The linear programming method is currently one of the most widely-used programming methods in social life. With a strong applicability and better effect, it is an effective means for reasonable allocation of funds, personnel and supplies.

\section{CORRELATION THEORY OF LINEAR PRO- GRAMMING AND MODELING}

The linear programming is a calculation method to research the optimization of the objective function in 


\section{MATEC Web of Conferences}

the operational research under the relevant linear restrictive conditions. The linear programming is relatively mature and complete in the operational research, and it especially occupies a very important position in the economic field. The linear programming has clear steps and little computing complexity, which is helpful for dealing with the problem quickly and very suitable for the economic field.

(1) Linear programming problem

In the problem, there is a set of related data values, and the data meets actual restrictive conditions. The restrictive conditions may be expressed by the linear equation or linear inequality. In addition, the related data values are related influencing factors of the objective function. There is a linear relation between them, so as to make the objective function achieve optimal results. This problem is called as the linear programming ${ }^{[3]}$.

Expressions of the linear programming:

Objective function:

$\max (\min ) z=C_{1} X_{1}+C_{2} X_{2}+\cdots+C_{n} X_{n}$

Meet the restrictive conditions:

$a_{11} x_{1}+a_{12} x_{2}+\cdots+a_{1 n} x_{n} \leq(\geq) b_{1}$

$a_{21} x_{1}+a_{22} x_{2}+\cdots+a_{2 n} x_{n} \leq(\geq) b_{2}$

$a_{m 1} x_{1}+a_{m 2} x_{2}+\cdots+a_{m n} x_{n} \leq(\geq) b_{m}$

Non-negative constraint conditions of the variables:

$x_{1}, x_{2}, \cdots x_{n} \geq 0$

The value of a set of $x_{1}, x_{2}, \cdots x_{n}$ that meets the restrictive conditions and non-negative constraint conditions of the variables is called as a feasible solution of the linear programming; when the value of a set of $x_{1}, x_{2}, \cdots x_{n}$ that meets the restrictive conditions and non-negative constraint conditions of the variables and the objective function achieves the optimal solution, it is called as the optimal solution of the linear programming ${ }^{[4]}$.

To transform the real problems into linear programming problems can express the impact of the various factors in the problems on the overall results, which can not only express the restrictive conditions well, but also determine the optimal solution of the objective result under such restrictive conditions. It requires accurately and actually transforming the problems, and determining the restriction factors and ultimate goals.

(2) Dual linear programming problem

There is often a correlation between many factors due to practical problems, and sometimes there is also a correlation between two results. The dual linear programming problem is actually the research of two related linear programming problems, so as to achieve the discussion of two problems, and obtain more practical solutions to two related problems ${ }^{[5]}$

Linear programming problem (I):

$\min z=C_{1} X_{1}+C_{2} X_{2}+\cdots+C_{n} X_{n}$

$a_{11} x_{1}+a_{12} x_{2}+\cdots+a_{1 n} x_{n} \leq(\geq) b_{1}$

$a_{m 1} x_{1}+a_{m 2} x_{2}+\cdots+a_{m n} x_{n} \leq(\geq) b_{m}$

$x_{1}, x_{2}, \cdots x_{n} \geq 0$

Linear programming problem (II):

$\operatorname{Maxg}=b_{1} y_{1}+b_{2} y_{2}+\cdots+b_{m} y_{m} \leq c_{1}$

$a_{11} y_{1}+a_{21} y_{2}+\cdots+a_{m 1} y_{m} \leq c_{1}$

$a_{12} y_{1}+a_{22} y_{2}+\cdots+a_{m 2} y_{m} \leq c_{2}$

$a_{1 n} y_{1}+a_{2 n} y_{2}+\cdots+a_{m n} y_{m} \leq c_{m}$

$y_{1}, y_{2}, \cdots y_{m} \geq 0$

The linear problem (II) is called as a dual problem of the linear problem (I).

(3) Shadow price ${ }^{[6]}$

In the dual linear programming problem, the shadow price is an optimal solution of the objective function obtained under the corresponding restrictive conditions. The shadow price is a valuation under the estimated situation. Through the shadow price, the enterprise can control over the cost well, so as to achieve a reasonable allocation of resources under a variety of conditions and improve planning capabilities.

The linear programming is a mathematical model widely used in the actual economic field in the operational research, which is an important means to solve the allocation of production resources, production planning and prediction of results, with a strong operability, a wide applicability, a high accuracy and many other advantages. It has successful applications in the improvement of enterprise management level and in the field of enterprise cost control. trol

(A) Objective function of the enterprise cost con-

The control of the enterprise cost is to obtain the optimal results within the most reasonable cost range and under the situation of resource utilization. The enterprise cost control model based on the linear programming is established to achieve the lowest cost and the largest objective result ${ }^{[7]}$. The objective function 
is:

$\max Z=C X$

Where: $C=\left(c_{1}, c_{2}, \cdots, c_{n}\right)^{T}$ is the product profit volume;

$c_{j}=p_{j}-c_{j}$ is the product profit;

$X=\left(x_{1}, x_{2}, \cdots, x_{n}\right)^{T}$ is the product vector.

(B) Constraint conditions of the enterprise cost control

The labor of enterprise production, equipment, raw materials, output and sales volume are restrictive conditions of cost limitation. The restrictive conditions have different degrees of impact on the objective results, so each restrictive condition has its own linear relation.

1. Enterprise labor costs:

$a_{11} x_{1}+a_{12} x_{2}+\cdots+a_{1 n} x_{n} \leq A^{\prime}$

Where: $a_{11}, a_{12}, \cdots, a_{1 n}$ are the enterprise labor costs caused by production of different products; $A^{\prime}$ is the enterprise labor costs payable.

2. Constraint conditions of raw materials:

$D X \leq D^{\prime}$

Where: $D=\left[\begin{array}{cccc}d_{11} & d_{12} & \cdots & d_{1 n} \\ \cdots & \cdots & \cdots & \cdots \\ \cdots & \cdots & \cdots & \cdots \\ d_{r 1} & d_{r 2} & \cdots & d_{r n}\end{array}\right]$ is the quantity demand of raw materials for the enterprise;

$d_{i j}$ is the quantity of the raw material $i$ required by the production of the product $j$;

$D^{\prime}=\left(d_{1}^{\prime}, d_{2}^{\prime}, \cdots, d_{m}^{\prime}\right)$ is the quantity of all raw materials required to be consumed;

$d_{i}^{\prime}$ is all the inventory of the raw material $i$

$r$ is all kinds of raw materials required by the enterprise production.

3. Production capacity:

$T X \leq T^{\prime}$

Where: $D=\left[\begin{array}{cccc}t_{11} & t_{12} & \cdots & t_{1 n} \\ \cdots & \cdots & \cdots & \cdots \\ \cdots & \cdots & \cdots & \cdots \\ t_{r 1} & t_{r 2} & \cdots & t_{r n}\end{array}\right]$ is the coeffi-

cient matrix of lost time in the enterprise product production process ${ }^{[8]}$;

$t_{i j}$ is the lost time of the process $i$ consumed by the production of the product $j$;
$T^{\prime}=\left(t_{1}^{\prime}, t_{2}^{\prime}, \cdots, t_{m}^{\prime}\right)$ is the time quantum required in each process;

'

$t_{i}$ is the time for the process $i$;

$r$ is the total quantity of the process.

4. Market demand:

$X \leq S$

Where: $S=\left(s_{1}, s_{2}, \cdots, s_{n}\right)^{T}$ is the market demand for the product.

3. Collection of the enterprise products production cost

The composition of the enterprise products production cost $(C)$ is divided into the raw materials consumption cost $\left(C_{1}\right)$, consumables cost $\left(C_{2}\right)$, production cost $\left(C_{3}\right)$ and labor cost $\left(C_{4}\right)$.

Enterprise products production cost:

$C=C_{1}+C_{2}+C_{3}+C_{4}$

Where: $C_{1}=\sum_{1}^{n} c_{1 i} x_{i} \quad c_{1 i}$ is the raw materials consumption cost in the process $i ; x_{i}$ is the usage amount;

$$
C_{2}=\sum_{1}^{n} c_{2 i} \bullet X \quad c_{2 i} \text { is the consumables cost in }
$$

the process $i ; X$ is the output;

$$
C_{3}=\sum_{1}^{n} c_{3 i} \bullet X \quad c_{3 i} \text { is the production cost in }
$$

the process $i ; X$ is the output;

$$
C_{4}=\sum_{1}^{n} c_{4 i} \bullet X \quad c_{4 i} \text { is the labor cost in the }
$$

process $i ; X$ is the output;

$$
c_{1 i}, c_{2 i}, c_{3 i} \text { and } c_{4 i} \text { can be calculated by the }
$$

following ways:

$c_{1 i}=P_{i}$ is the price of the produced product $i$;

$c_{2 i}=\frac{\sum C_{e i} \bullet P_{e}}{\sum X}: \sum X_{e i}$ is the consumables cost in the process $i ; P_{e}$ is the price of consumables; $\sum X$ is the output;

$c_{3 i}=\frac{\sum C_{m i}}{\sum X}: \sum C_{m i}$ is the production cost in the process $i ; \sum X$ is the output;

$c_{4 i}=\frac{\sum C_{s i}}{\sum X}: \sum C_{s i}$ is the labor cost in the process 


\section{MATEC Web of Conferences}

\section{$i$; $\sum X$ is the output;}

The restrictive conditions of the objective function (cost control) can be found out by the expression of the enterprise cost structure, so as to express the objective function well. The linear relation of the objective function can be found out through the restrictive conditions, so as to achieve the purpose of the linear programming ${ }^{[9]}$.

\section{MODEL SOLUTION}

There are many influencing factors of the enterprise cost control, so the calculated quantity of the model solution will be increased in case of practical problems. Therefore, the use of computer to calculate can speed up the problem solving, and play a role of fast solving, and reducing complex calculation by manpower. There is a need to firstly determine whether there is a basic feasible solution of the cost control model based on the linear programming, and then calculate the optimal results.

Algorithm flow chart is as Figure 1.

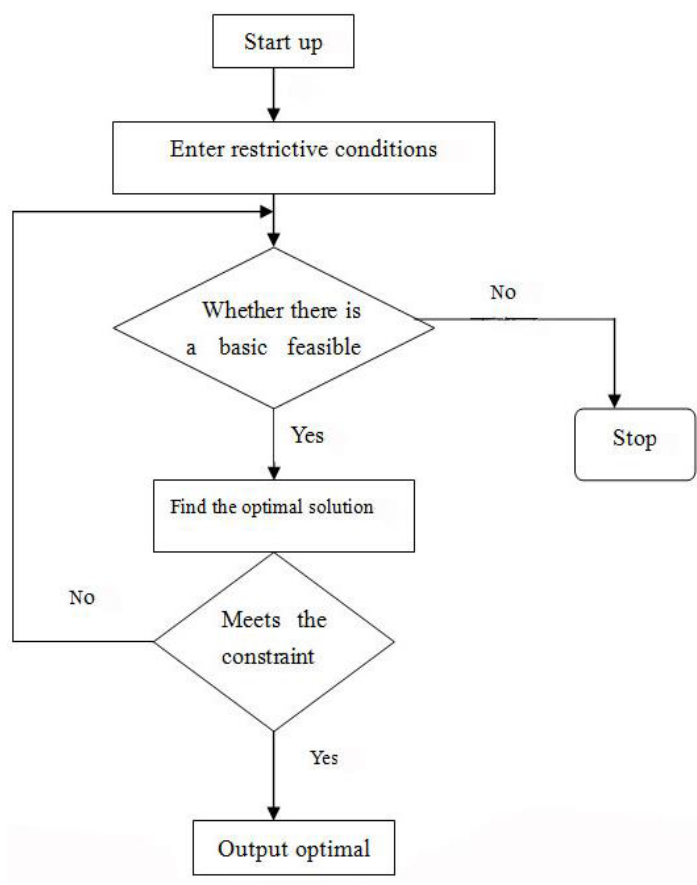

Figure 1. Algorithm flow chart

\section{CASE APPLICATION}

The case application can be used to test the established enterprise cost control model based on the linear programming. In the production plan of an enterprise and according to preliminary market research, three kinds of products are ready to be produced. Each commodity's production labor costs, raw materials costs, number of equipment units, profit of single product are shown in Table 1. We would like to maximize the use of raw materials to achieve maximum enterprise efficiency with the limited labor costs. This is a typical linear programming problem.

Under the premise of limited productive resources, it plans to use the lowest cost to achieve maximum benefits. The constraint conditions are the labor of production, raw materials, equipment and so on. The objective function is the maximum income ${ }^{[10]}$. To substitute these conditions into the established minimum enterprise cost control model based on the linear programming, the model solution is:

$$
\begin{gathered}
\max Z=3 x_{1}+7 x_{2}+8 x_{3} \\
\left\{\begin{array}{c}
100 x_{1}+400 x_{2}+300 x_{3} \leq 45000 \\
6 x_{1}+2 x_{2}+5 x_{3} \leq 180 \\
5 x_{1}+4 x_{2}+10 x_{3} \leq 180 \\
x_{1} \geq 0, x_{2} \geq 0, x_{3} \geq 0
\end{array}\right.
\end{gathered}
$$

The relevant enterprise production plans can be developed, and the existing production resources can be rationally used by the enterprise through the calculation results of the enterprise cost control model based on the linear programming, so as to achieve the maximum enterprise profit at the lowest cost. The market is constantly changed, so it is necessary to timely modify the model to achieve constant adjustment of the production and ensure the maximization of enterprise profits, thereby providing guarantee for the enterprise to maintain a strong competitiveness in the fierce market competition.

\section{SENSITIVITY ANALYSIS}

The sensitivity analysis is for the stability of the model, mainly inspecting the overall change degree of the model in case of changes of the related factors. In the actual market environment, the price, raw materials,

Table 1. Products production table

\begin{tabular}{l|llll}
\hline Product & Product 1 & Product 2 & Product 3 & \\
\hline Unit profit & 3 & 7 & 8 & Unit (ten thousand yuan) \\
Labor costs & 100 & 400 & 300 & Total $(45,000)$ \\
Raw material & 6 & 2 & 5 & $180 \mathrm{t}$ \\
\hline Number of equipment units & 5 & 4 & 10 & 90 units \\
\hline
\end{tabular}


labor and equipment are constantly changed, so there is a need of dynamic adjustment of the enterprise products production management and plan arrangement, so as to maintain continuously maximum profits of the production enterprise.

Price change of the products produced by the enterprise

The price change of the products produced by the enterprise will lead to changes of the enterprise profits, which can be calculated by the sensitivity test formula:

$$
\delta_{j}=C_{j}-C_{B} B^{-1} P_{j}
$$

From the formula, the price change will affect the number of tests. In order to achieve the optimal solution of the objective function, there is a need to adjust the production arrangement.

\section{CONCLUSION}

In the enterprise cost control problem, the use of linear programming model is one of the most widely-used and effective ways, which can not only calculate the optimal profit, but also guide the specific production planning. The shadow price in the linear programming is to analyze the influencing factors. The degrees of impact of different restrictive factors on the objective function can be calculated by the shadow price, thereby making targeted adjustment to achieve maximum enterprise production efficiency and income.

This paper researches the enterprise cost control through the linear programming model, and analyzes the restriction factors of the labor of enterprise production, raw materials, processing equipment, sales price, and other factors affecting the enterprise income, so as to obtain an enterprise cost control model based on the linear programming. This model can calculate rational production mode in the case of limited resources, and achieve optimal enterprise income. The production guiding program and scheduling arrangement of the enterprise can be obtained through calculation results, so as to provide scientific and effective guidance for the enterprise production. This paper adds the sensitivity analysis in the linear programming model, so as to learn about the stability of the enterprise cost control model based on linear programming through the sensitivity analysis, and verify the rationality of the model.

With a simple structure, the enterprise cost control model based on the linear programming includes all major influencing factors. With a simple calculation, it can accurately obtain the optimal result of the objective function and plan arrangement. Therefore, this model is characterized by simplicity and high efficiency, which can better adapt to the new enterprise planning situation under the market changes. It provides a certain reference for the enterprise planning in the market economy environment, which has strong reference and practical significance in terms of the enterprise cost control.

\section{ACKNOWLEDGEMENT}

This paper is financially supported by the National Vocational Standard-oriented Reform and Research of Higher Vocational Accounting Courses (No. PJHYZD15031) and Practice and the Research of Higher Vocational Accounting Computerization Courses Reform (No. LZY15562).

\section{REFERENCES}

[1] Huang Guidong, \& Ding Wenying. 2006. Research on optimization of production plan based on linear programming. Logistics Technology, (2): 83 - 85.

[2] Wu Shuhe. 2009. Calculation and economic analysis of shadow price. Journal of Changchun University of Technology (Social Sciences), 9 (3): 75-79.

[3] Tian Guoqiang. 2000. Characterizations of Fixed-Point Theorems, Optimization, and General Equilibria. Peking University Press.

[4] Hu Yunquan. 2004. Basis and application of operational research. Beijing: Tsinghua University Press.

[5] Sun Tingfeng. 2006. Brief analysis of linear programming application in the enterprise production plan. Business Economics, (276): 18 - 20.

[6] Zhao Maoxian, \& Gao Muyou. 2005. Global optimization method for initial bilevel linear programming. Journal of Operational Research, (2).

[7] Pan Pingqi, Li Wei, \& Wang Yong. 2004. Dual simplex algorithm I of deficient basis based on the most obtuse rule. Journal of Operational Research, (2).

[8] Niu Li, \& Zhai Xiuna. Applied research on the linear programming theory in enterprise management. Market Modernization, (472): 195-196.

[9] Zhou Zhifang, \& Xiao Xu. Research on construction of resource value flow models of process manufacturing enterprises. Journal of China University of Geosciences (Social Sciences): 43-50

[10]Barfield Kinney. 2003. Cost Accounting: traditions and innovation CMS. 40-52 